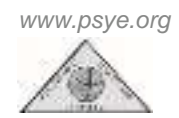

\title{
Una propuesta de intervención para prevenir el acoso escolar desde una perspectiva sociogrupal
}

\author{
Esther Noemí Leganés Lavall* \\ Universitat Jaume I de Castellón
}

(Recibido 14 de Febrero 2012;Aceptado 13 de Septiembre 2012)

RESUMEN: En este estudio se presenta una propuesta de intervención para prevenir el acoso escolar desde una perspectiva sociogrupal. Tras analizar diferentes informes españoles sobre convivencia escolar, se observa que el apoyo a la víctima por parte del grupo de espectadores resulta ser una de las medidas más eficaces. Sin embargo, en la mayoría de los casos los espectadores muestran actitudes pasivas ante la agresión o incluso llegan a considerarla divertida. Por ello se elabora un programa que trabaja la conducta prosocial a través de su componente principal: la empatía. Los datos presentados por diferentes programas españoles en relación a la convivencia en el aula, muestran la importancia de seguir llevando a cabo intervenciones orientadas especialmente en la prevención, para erradicar las conductas abusivas antes de que éstas aparezcan.

Palabras clave: educación primaria, conducta prosocial, espectadores, empatía.

\section{An intervention proposal to prevent bullying from a sociological perspective}

\begin{abstract}
This study introduces an intervention proposal to prevent bullying from a socio-group view. After analyzing different Spanish reports on school life, it is shown that the support by the peer group to the victim is one of the most effective actions. However, in most cases peer group show passive attitudes to aggression or even they consider it fun. Thus, here is developed a program that works prosocial behavior through its main component: the empathy. Data presented by different Spanish programs in relation to school life, show the importance of carrying out further interventions, emphasizing prevention to eradicate abusive behavior before it arises.
\end{abstract}

Keywords: elementary education, prosocial behavior, onlooker, empathy.

\section{INTRODUCCIÓN}

El acoso escolar 
El concepto de acoso escolar cuenta con multitud de definiciones, desde que en la década de los 70 se convirtiera en el centro de atención de la psicología y comenzaran a realizarse los primeros estudios. Inicialmente, las investigaciones utilizaron diversidad de criterios para evaluar la incidencia del acoso entre iguales tales como los roles de los alumnos implicados, los tipos de agresión o

\section{*Correspondencia: Esther Noemí Leganés Lavall, Universitat Jaume I de Castellón. E-mail: esthernoemi@hotmail.com}

los factores que inciden en ella (Górriz, 2009). El auge y consolidación de los estudios sobre acoso escolar se da entre la década de los 80 y de los 90 propagándose a países como Gran Bretaña, Noruega, Países Bajos, Japón y España (Gómez, Gala, Lupiani, Bernalte, Miret, Lupiani, Barreto, 2007). Más tarde, este fenómeno se expande a nivel internacional, desarrollándose estudios alrededor de diferentes focos de atención tales como familias, género y grupo de escolares, entre otros (Mora-Merchán, 2000).

El acoso escolar, también conocido como maltrato entre iguales o bajo el término en inglés "bullying” acuñado por Dan Olweus, es un tipo específico de violencia que viene caracterizado por comportamientos violentos reiterados sobre la misma persona, agresiones a nivel físico y psicológico, desigualdad entre el agresor y la víctima, y la intencionalidad del agresor de hacer daño (Ortega, 1999; Avilés, 2006; Serrano, 2006). Estos aspectos resultan alarmantes ya que: "La continuidad de estas relaciones [víctima-agresor] provoca en las víctimas efectos claramente negativos: ansiedad, descenso de la autoestima, y cuadros depresivos, que dificultan su integración en el medio escolar y el desarrollo normal de los aprendizajes" (Olweus, 1998, p.25). Es decir, la víctima resulta afectada en su desarrollo social, psicológico, biológico y afectivo, hecho que refleja también la Organización Mundial de la Salud al definir cualquier acto de violencia en general como: "El uso intencional de la fuerza o el poder físico, de hecho o como amenaza, contra uno mismo, otra persona o un grupo de comunidad, que cause o tenga muchas probabilidades de causar lesiones, muerte, daños psicológicos, trastornos del desarrollo o privaciones" (Organización Mundial de la Salud, 2003, p.5). Si tal y como indica la OMS, cualquier acto violento puede desencadenar graves daños sobre aquellos que la sufren, y: "La violencia escolar ha sido reconocida como un problema creciente y destructivo tanto por las autoridades educativas en un nivel nacional, como por la Comisión Europea a un nivel europeo" (Staneva y Molhova, 2011, p.147), cuanto más el acoso escolar por su carácter reiterado y prolongado en el tiempo, puede desencadenar consecuencias irreversibles tanto en víctimas como agresores.

Analizando los estudios realizados en los últimos años a nivel internacional, se encuentra el informe "Health behavior in school-aged children" (Currie et al., 2008), que en su sección 4 estudia las conductas de riesgo entre las cuales se encuentra el "bullying". Examinando las cifras en niños de educación Primaria (11 años) se observa que en nuestro país, un $11 \%$ de los participantes afirmaron haber sufrido acoso escolar en el año 2006 (p. 160), datos que resultan 
alentadores frente al 53,8\% que este mismo estudio revelaba en el año 2002 como muestra la siguiente gráfica (Currie et al., 2004):

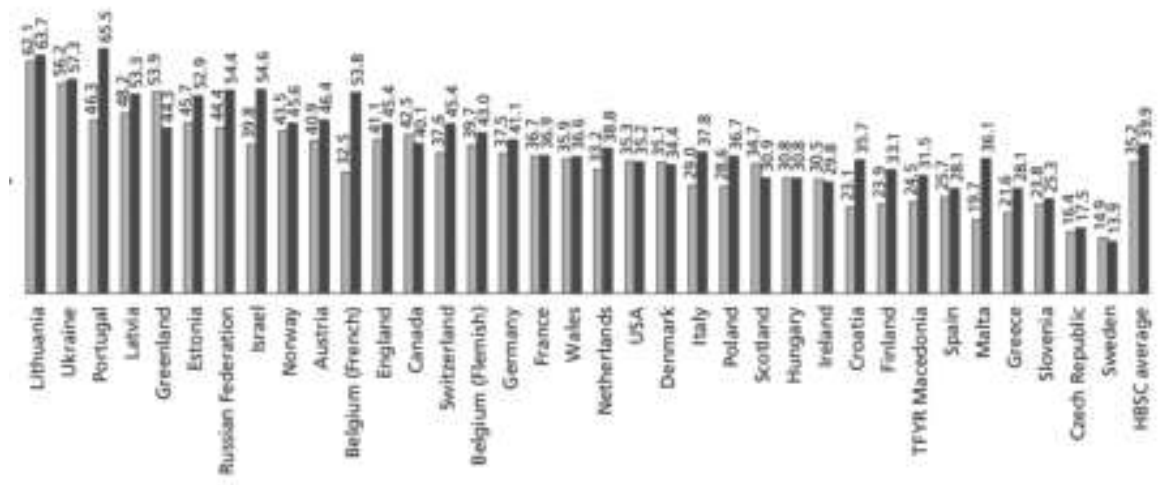

Figura 1. Datos del informe "Health behavior in School-aged children" (2002).

Esto es gracias a que, tal y como señalan Ortega, Romera y del Rey (2009, p. $5)$ :

En España, desde principios de la década de los noventa, no han dejado de diseñarse y desarrollarse múltiples y variadas iniciativas encaminadas a hacer frente al problema de la violencia escolar. Los distintos programas y planes desarrollados dentro las comunidades autónomas han logrado ofrecer un importante acervo de propuestas innovadoras que centran sus esfuerzos en superar la conflictividad y en avanzar hacia la conquista de una vida democrática dentro y fuera de las escuelas.

De aquí que puedan encontrarse diversos estudios e informes que examinan la convivencia escolar en los centros educativos (Avilés y Monjas, 2005; Bolado y Gómez, 2005; Gómez-Bahíllo, 2006; Jiménez, 2007; Sáinz, Angulo, Elorza, y Méndez, 2008; Iborra, Rodríguez, Serrano y Martínez, 2011). En ellos, vienen reflejadas diferentes tipos de conductas violentas que afectan de una u otra forma a la convivencia escolar, aspectos tales como los lugares donde ocurre el acoso escolar, las formas en que éste se manifiesta, sus causas y consecuencias..., siendo uno de los más relevantes: "en qué medida aparecen los diferentes tipos de violencia escolar evaluados [...] para establecer líneas de intervención ajustadas a la realidad actual de los centros educativos" (González, Álvarez, Rodríguez, González, Núñez, Álvarez, 2010, p.119). Otro aspecto importante son los participantes en el acoso escolar, ya que además de víctimas y agresores, encontramos una serie de agentes cuya implicación se da tanto de forma directa como indirecta. Christina Salmivalli (1999), señala que el acoso escolar ocurre generalmente en situaciones donde muchos miembros del grupo de iguales están presentes, y por ello incide en la importancia que tienen los espectadores, quienes tomarán un rol u otro en función de factores personales y contextuales, como por ejemplo, el estatus social que presentan en el grupo: rechazado, ignorado, 
promedio, popular y controvertido (Salmivalli, Lagerspetz, Björkqvist, Österman, kaukiainen, 1996).

En los últimos años, y a través de los esfuerzos de muchos investigadores preocupados por este asunto, han surgido importantes avances en la intervención sobre el acoso escolar a nivel internacional. Un ejemplo de ello es la Federación Internacional de investigadores en el campo de la violencia en las escuelas, iniciada en 1998 por iniciativa del "Observatorio Europeo de la Violencia Escolar". Tras continuas reuniones internacionales y el progresivo crecimiento de nuevos proyectos se establece el "Observatorio Internacional de la Violencia en la escuela".

\section{LA CONDUCTA PROSOCIAL}

\section{El papel de los iguales}

En las situaciones de acoso escolar, el rol de los iguales ha sido explicado desde diferentes perspectivas teóricas como el enfoque cognitivo-evolutivo, la perspectiva etológica y las teorías del aprendizaje, cuyo eje de partida se constituye en la importancia que tienen los iguales para desarrollar las competencias sociales. En los últimos años se ha producido un auge de intervenciones que se centran en el trabajo con el grupo de iguales (del Barrio, Barrios, Granizo, van der Meulen, Andrés y Gutiérrez, 2011). Es por ello que la propuesta aquí presentada se fundamenta en el estudio del enfoque sociogrupal, el cual parte de la base de los distintos niveles de poder existentes dentro de cada uno de los grupos sociales, bien por género, identidad cultural o clase social (Górriz, 2009).

En el enfoque sociogrupal, el grupo de iguales juega un importante papel como transmisor de normas culturales. En los casos de acoso escolar, diversos estudios como los llevados a cabo por autores como Patterson, Shaw, y Ebner (1969); Solomon y Wahler (1973); Patterson, Littman y Bricker (1967), demostraron ya en la década de los 60 y 70, que las conductas de los agresores se refuerzan por parte de los compañeros, quienes mayormente juegan el rol de espectador en los casos de acoso escolar. Esta idea ha llevado a estudiar los refuerzos y modelos positivos que pueden desprenderse de la influencia de los iguales, para mantener conductas deseables como la conducta prosocial. Aunque existen discrepancias en el mundo científico sobre la definición de conducta prosocial (Guijo, 2002), Eisenberg y Fabes (1998) tras llevar a cabo una de las revisiones más completas sobre este tema, la definen como:

[...] voluntaria y con intención de beneficiar a otros. [...] determinada por múltiples causas, incluido egoísmo, orientación hacia los otros e intereses concretos. Especial importancia para entender el razonamiento moral es el subgrupo de conductas prosociales denominadas altruismo (Eisenberg y Fabes, 1998, p.702). 
Diferentes autores han tratado de categorizar la conducta prosocial (González Portal, 1992; Masnou, 1994; Eisenberg y Fabes, 1998; Greener y Crick, 1999). Entre estas categorías encontramos: ayuda instrumental, ayuda no instrumental, consolar, confortar, compartir, donar, cooperar, ayuda en situación de emergencia y defender. Para la evaluación de la conducta prosocial, encontramos los siguientes instrumentos (Garaigordobil, 2005): Weir y Duveen (1981) elaboran un cuestionario de conducta prosocial. Contiene 20 ítems de conductas prosociales en relación a las cuales el profesor/a informa de las conductas que observa en sus alumnos/as; Mehrabian y Epstein (1972) crean un cuestionario de evaluación de la empatía. Consta de 22 frases que hacen referencia a sentimientos empáticos con los que se mide la capacidad de empatía. La tarea consiste en leer un conjunto de afirmaciones indicando si habitualmente hace, piensa o siente lo que afirma la frase; Ma y Leung, 1991, presentan un autoinforme que consta de 24 afirmaciones referidas a comportamientos altruistas y sentimientos empáticos, base de la conducta prosocial, con los que se mide la orientación altruista del individuo. La tarea consiste en leer las afirmaciones indicando si habitualmente se hace, piensa o siente lo que afirma la frase.

La conducta prosocial puede ser medida en base a una serie de variables, entre las que destaca la empatía como su componente básico. Hoffman (2000) la define como la respuesta afectiva más congruente con la situación de otra persona que con la propia, es decir, la capacidad de ponerse en el lugar del otro. La empatía está formada por componentes afectivos y cognitivos, a través de los cuales una persona puede sentir y entender lo que está sintiendo otra persona. En función del grado de altruismo, podemos hablar de empatía disposicional y situacional, siendo la primera un rasgo de personalidad y por lo tanto de mayor estabilidad empática, mientras que la segunda se refiere al grado afectivo ante una situación concreta y depende de unas variables contextuales (Fuentes, 2005).

\section{MéTODO}

\section{Objetivos}

Los objetivos de este estudio fueron en primer lugar, conocer el actual estado de la cuestión sobre la intervención en el acoso escolar. En segundo lugar, elaborar una propuesta en base a las directrices de las últimas investigaciones.

\section{Diseño}

Para llevar a cabo los objetivos propuestos, se realizó una revisión sobre el estado de la cuestión de las intervenciones realizadas en los últimos años (del Rey y Ortega, 2001; Avilés, 2003; Andrés y Barrios, 2006; Díaz-Aguado, 2006a; Díaz-Aguado, 2006b; Andrés y Barrios, 2008; del Barrio et al., 2011), y junto con el análisis de recientes programas de convivencia a nivel estatal (Serrano e Iborra, 2005; Defensor del pueblo y UNICEF 2006), se elaboró una propuesta formada por una temporalización de actividades que trabajan transversalmente la 
conducta prosocial a través de su variable fundamental: la empatía, como competencia social y herramienta de mejora de la convivencia escolar, entendida ésta como "prevención de la violencia escolar" (del Rey y Ortega, 2001, p. 143).

\section{Participantes}

Esta propuesta de programa está dirigida para ser implementada en el segundo ciclo de Primaria, más concretamente en el aula de $3^{\circ}$, cuyos alumnos se encuentran en el rango de edad de entre los 8 y 9 años.

\section{Instrumentos}

Para la recogida de datos y posterior elaboración de esta propuesta de intervención, se confeccionó una plantilla ad hoc que recopila la información aportada por investigaciones, programas diseñados para mejorar la convivencia escolar en los centros educativos españoles, además de diversos materiales y recursos existentes en torno al tema. Cada actividad se recogió en una plantilla, formando así un conjunto de fichas con información para la elaboración de esta propuesta. 
Tabla1. Plantilla para la Recogida de Actividades

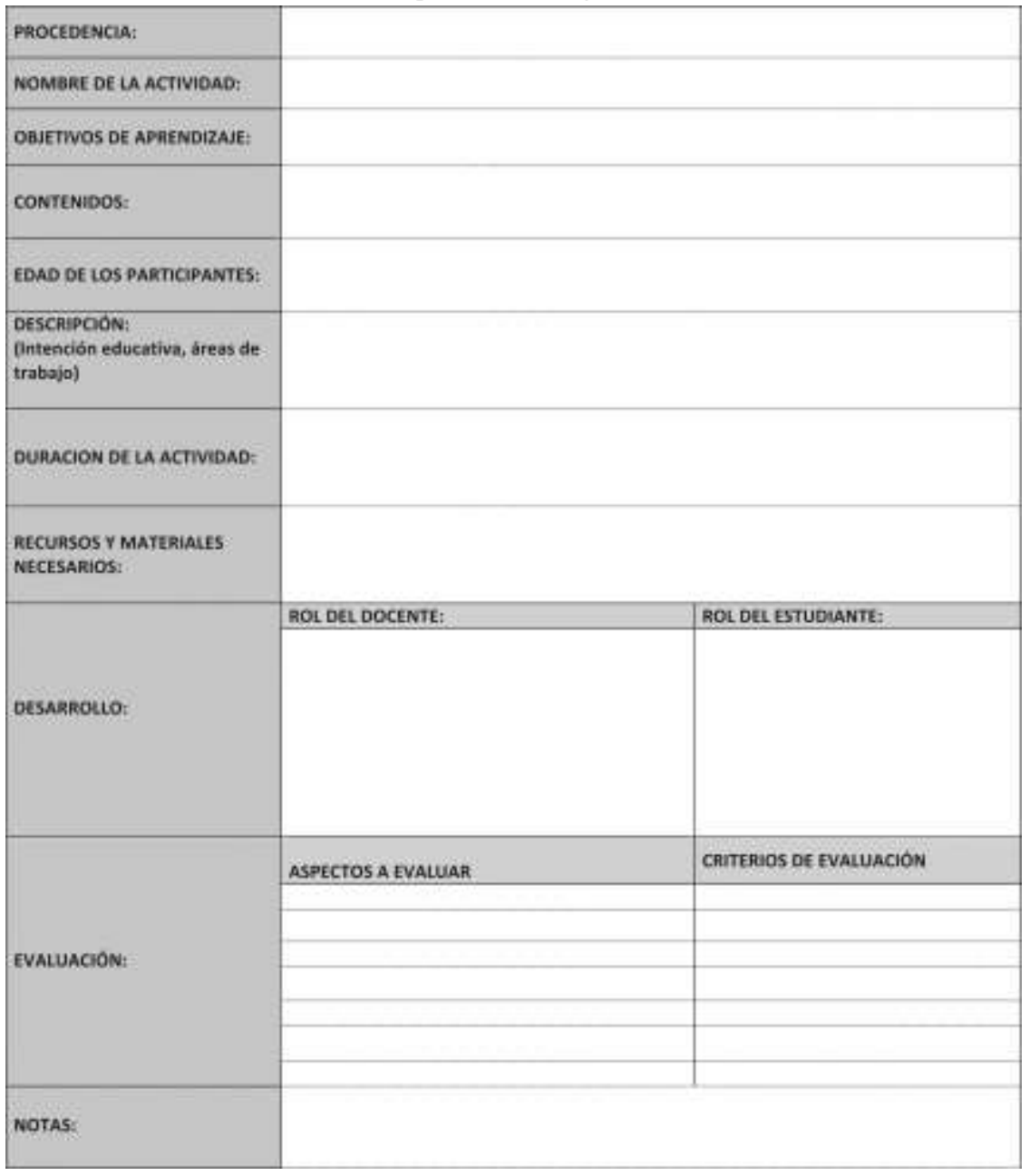

\section{Procedimiento}

El procedimiento seguido se divide en las siguientes fases:

- Fase I. Recopilación de investigaciones y programas de prevenciónintervención en la convivencia escolar.

- Fase II. Revisión y análisis de las investigaciones y programas, y recolección en la plantilla de actividades relacionadas con el trabajo de la conducta prosocial desde la empatía.

- Fase III. Elaboración de una propuesta para trabajar la conducta prosocial.

- Fase IV. Futura implementación del programa.

$$
\text { PROPUESTA DE INTERVENCIÓN }
$$


Partiendo de la concepción sobre el acoso escolar como problema social, y de la existencia de numerosos programas de prevención e intervención, se plantea la presente propuesta teniendo en cuenta que: "Algunas de estas sugerencias y planteamientos parecen mal enfocados o incluso contraproducentes, tales como centrarse en exceso en el hecho de cambiar el comportamiento de las víctimas para hacerlas menos vulnerables al acoso escolar" (Olweus, 2001, p.16). Es por ello, que al examinar las diferentes vertientes desde las cuales puede ser trabajado el acoso escolar, se considera orientar esta propuesta a modificar, principalmente, el rol del espectador, mediante el trabajo de la conducta prosocial a través de su variable empatía. El proyecto se ubica dentro del apartado de propuestas de aula puesto que se trata de una acción planificada y fundamentada en la prevención, y está construido sobre los cinco pasos de Latané y Darley (1970):
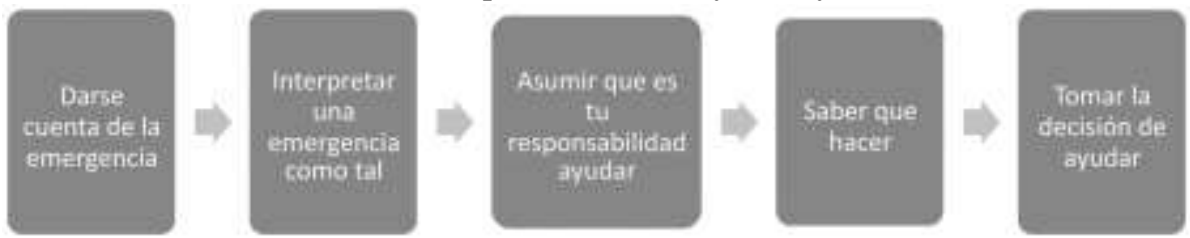

Figura 2. Proceso de toma de decisiones según Latané y Darley (1970).

El motivo por el cual se ha decidido trabajar con alumnado de $3^{\circ}$ de educación Primaria, es porque según la Teoría de las Etapas de Desarrollo Cognitivo de Piaget (1997), estos alumnos se sitúan en la "Etapa de las Operaciones Concretas", donde los procesos de razonamiento se vuelen lógicos y pueden aplicarse a problemas concretos o reales. En el aspecto social, el niño ahora se convierte en un ser verdaderamente social y en esta etapa aparecen los esquemas lógicos de seriación, ordenación mental de conjuntos y clasificación de los conceptos de casualidad, espacio, tiempo y velocidad. "Esto nos ayuda a trabajar las actividades grupales y de forma cooperativa, y a potenciar la participación activa de los alumnos" (Leganés y Pérez-Aldeguer, 2012, p.116).

\section{Desarrollo del proyecto}

La metodología empleada para desarrollar el programa será activa y creativa, de forma que ayude al alumnado a desarrollar una conducta prosocial. Se llevará a cabo mediante el trabajo grupal, mediante las siguientes estrategias didácticas:

1. Estrategias de aproximación a la realidad. Se facilitará al alumnado la posibilidad de observación, experimentación y análisis de situaciones reales. Para ello, se realizarán actividades tales como dramatizaciones y resolución de dilemas morales.

2. Estrategias de descubrimiento. Utilizando ilustraciones, videos, imágenes o cualquier material visual, el docente por medio de preguntas guiará al alumno a identificar situaciones de acoso escolar. 
3. Estrategias de problematización. Se expondrán problemas reales por medio de actividades como historias y role-playing donde el alumnado tendrá que buscar y consensuar posibles soluciones.

4. Estrategias de trabajo colaborativo. Todas las actividades serán colaborativas, con lo cual se estará trabajando la conducta prosocial.

El programa propuesto, permite ser llevado a la praxis desde dos formas diferentes pero siempre en relación al curriculum. En primer lugar, relacionando las actividades directamente con el contenido de la asignatura, por ejemplo, en clase de Lengua Castellana, se realiza una actividad donde se trabaje la empatía por medio de la lecto-escritura. En segundo lugar, dedicando parte de la clase al desarrollo de la asignatura y la otra parte a una actividad del programa. Esta propuesta está planteada para ser implementada de forma transversal y alternada con el trabajo de las asignaturas del aula de $3^{\circ}$ de Primaria. Consta de una actividad y su posterior debate y reflexión, siempre acorde con el contenido de la asignatura y aumentando el grado de dificultad progresivamente. En ningún momento, se hace alusión en las actividades a los términos: víctima, agresor, para no reforzar su existencia. Este programa está diseñado para trabajarlo a lo largo del primer cuatrimestre del curso, el cual está formado por 15 semanas aproximadamente. Cada semana se trabajarán las actividades en 2 sesiones consecutivas de una hora, y en asignaturas alternas. Por ejemplo: semana 1: Música y Lengua Castellana; semana 2: Matemáticas y Educación Física. Al cabo del trimestre, se habrán trabajado 30 sesiones. En este estudio se desarrolla una propuesta de actividades para trabajar la empatía, cada una, diseñada para ser implementada a lo largo de 1 hora con una introducción previa de 10 minutos donde se relacione el contenido de la actividad con el de la asignatura. Para finalizar, se realizará en todas las actividades unos minutos de reflexión y debate acerca de la actividad. A continuación, se muestra un ejemplo de actividades tipo diseñadas para el trabajo sociogrupal. Se han extraído ideas de diferentes programas de intervención así como de otros materiales en Internet, adaptándolas a los objetivos propuestos.

Tabla 2. Actividades de Muestra

\begin{tabular}{cc}
\hline SESIÓN & ACTIVIDAD \\
\hline 1 & ¿Hay algún problema? \\
2 & El león y el ratón \\
3 & †Tengo un dilema! \\
4 & Bully Dance \\
5 & Los dos monstruos \\
6 & Ponte en mi lugar \\
7 & Y tu iQué harias? \\
\hline
\end{tabular}

Tabla 3. Actividad 1. -¿Hay algún Problema? 
* Introducción temática: los problemas matemáticos.

- Trabajamos los pasos 1 y 2 de Latané y Darley para aprender a identificar una emergencia/problema.

- Desarrollo: exponemos diferentes historias y los alumnos identificarán si se trata de un problema o no, a través de las emociones que los protagonistas de las diferentes historias pueden estar sintiendo. Se trata de identificar los problemas a través de situaciones negativas.

Ejemplo de historia: (Modificado de ESCEPI)

1. Cuando sonó el timbre que anunciaba el recreo, Juan de 3er curso cogió su balón y bajó al patio a jugar un partido con 3 compañeros de clase. ¿Cuál creéis que seria su emoción?

Cuando estaban jugando dos chicos de $4^{\circ}$ curso les quitaron el balón y se lo llevaron para jugar con sus amigos. ¿Crećis que hay un problema? ¿Por quê? ¿Cómo creéis que se sentirán Juan y sus amigos?

Otra posibilidad es la de proyectar diferentes fragmentos de video o mostrar viñetas con situaciones donde el alumnado pueda identificar si existe un problema o no. Podemos realizar las siguientes preguntas:

- ¿Qué pasa en el video/ viñeta?

- Crees que se trata de un problema? iPor qué?

\section{Tabla 4. Actividad 2. -El León y el Ratón}

\section{ACTIVIDAD 2: EL LEÓN Y EL. RATOÓN}

- Introducción temática: los animales

- Trabajamos las percepciones y la conducta prosocial.

- Desarrollo: mostramos a los illumnos el video de la fábula del león y el ratón: http://www youtube,com/wutch?v=yAma4fFCOgl. Podemos hacer las siguientes preguntas:

- Qué es lo que ocurre en el video?

- ¿Qué habéis pensado cuando el ratón se acercaba al león? ¿Cuántos pensaban que se lo iba a comer? ¿Cuántos pensaban que le iba a ayudar?

- ¿Por qué le ha ayudado?

- ¿Qué hace el ratón cuando ve al león en apuros?

- Qué pasa al final?

\section{Tabla 5. Actividad 3. - ¡Tengo un Dilema!}

\section{ACTIVIDAD 3: -TENGO UN DILEMA:}

- Introducción temática: Ayudar a los demás.

- Trabajamos el paso 4 "Saber que hacer" a través de los dilemas morales.

- Desarrollo: Dividimos la clase en grupos y repartimos un dilema a cada grupo. A continuación dejamos un tiempo de lectura y debate. Mientras tanto, el docente resolverá las dudas de sus alumnos. Cuando finalicen, cada grupo leerá su dilema contestando a la pregunta final. Abriremos un debate 
de clase a través del planteamiento de más preguntas. Algunos ejemplos de dilemas:

Dilema l:

Juan va a la cafeteria durante el recreo a comprarse el almuerzo. En el camino ve cómo Sara, José y Pedro, unos compañeros de otro curso, se han llevado a un rincón a Antonio, un alumno de un curso anterior. Los tres le están amenazando para que Antonio les dé el dinero del almuerzo. Juan se acerca y les dice que le dejen en paz, pero los tres compañeros le dicen que se vaya y no se meta en un asunto que no es el suyo. Además le amenazan con pegarle una paliza si cuenta a alguien lo que ha visto,

¿Debe Juan denunciar lo ocurrido a algin profesor del Colegio?

Posibles preguntas para iniciar el debate del dilema 1:

- ¿Qué pasa en vuestra historia?

- ¿Cómo os sentiriais en el lugar de Antonio?

- ¿Creéis que está bien lo que hacen Sara, José y Pedro? ¿Por qué?

- ¿Qué hariais vosotros si fuerais Juan?

Dilema 2:

Marta lleva un año en el colegio y este año empieza el cuarto curso de Primaria. El año pasado no logró integrarse en ningún grupo de amigos. aunque lo intentó varias veces. Este año se ha propuesto formar parte del grupo en el que están las que mejor le caen. Cuando les pide que quiere pertenecer a su grupo, le contestan que para hacerlo debe ayudarles a gastar una broma a alumnos de segundo. Como novatada, proponen acercarse a alumnos de segundo en el patio y obligarles a que les den el bocadillo o el dinero para el bacadillo, con la amenaza de darles una paliza si no les obedecen.

¿Debe Marta participar en la novatada?

Posibles preguntas para iniciar el debate del dilema 2:

- ¿Qué pasa en vuestra historia?

- ¿Cómo os sentiriais en el lugar de Marta?

- Crecéis que están bien las novatadas? (explicar el significado si es necesario)

Tabla 6. Actividad 4. -Bully Dance

\section{ACTIVIDAD 4: BULLY DANCE}

(Actividad modificada de la Propuesta elaborada por Collell - Escudó)

- Introducción temática: la danza y los instrumentos de pequeña percusión.

- Trabajamos los comportamientos violentos.

- Desarrollo: pedimos a los alumnosias que cojan un folio y un lápiz, diciéndoles que vamos a ver el video "Bully Dance" sobre cosas que pasan en la escuela y que apunten aquellas cosas que les llaman la atención, fijándose bien en los detalles. También apuntarán los instrumentos de pequeña percusión que aparecen durante el video: bttp:/www youtube, com/watch? $v=$ jlB8kyphcM Cuando termine, les pedimos que digan lo que han entendido sobre la historia. Nos interesa enfatizar sobre el rol de la mayoria de las hormigas (espectadores), Podemos hacerlo a través de las siguientes preguntas:

- ¿Qué hacen la mayoria de las hormigas cuando la más grande se mete con la más pequeña? ¿Por qué ereéis se comportan asi?

- ¿Qué pensáis que deberian haber hecho las demás hormigas?

- ¿Cómo os sentiriais si fuerais la hormiga pequeña? ¿ $\mathrm{Y}$ si fuerais las 

que la historia acaba bien o mal y por qué.

- Finalizamos la actividad repitiendo la parte del video donde aparece la melodia de la danza final, y les pediremos a los alumnos que utilizando instrumentos de percusión que han visto en el video, representen la misma situación pero con un final alternativo donde todos acaban siendo amigos y danzando juntos.

Tabla 7. Actividad 5. -Los Dos Monstruos

\section{ACTIVIDAD 5: LOS DOS MONSTRUOS}

(Texto obtenido de David MeKee. Editorial: Espasa Calpe. Madrid)

- Introducción temática: El sol y la luna. La noche y el día.

- Trabajamos la agresividad verbal y los insultos.

- Desarrollo: pedimos tres voluntarios/as para leer un cuento de forma teatral. Los papeles son los siguientes:

Voluntario 1: Monstruo 1 (M1)

Voluntario 2: Monstruo 2

Voluntario 3: Narrador/a

Colocamos 2 sillas frente a la clase. Estas sillas están de espaldas la una de la otra y a una distancia aproximada de dos metros. En medio de las dos sillas se coloca un cartón alto o algún objeto que impida que se vean. Podemos simular unas piedras con papel de periódico arrugado esparcido por el suelo.

- Cuento:

Narrador/a: Erase una vez un monstruo que vivía tranquilamente en la ladera oeste de una montaña. En la ladera este de la montaña vivía otro monstruo. Los monstruos se hablaban a veces a través de un agujero de la montaña. Pero nunca se habían visto. Una tarde el primer monstruo dijo:

M1: ¿Has visto qué bonito? El día se marcha.

M2: ¿Que el día se marcha? ... ¡Querrás decir que la noche llega ceporro!

M1: ¡zopenco! ¡No me llames ceporro que me enfado!

$\mathrm{N}$ : y se quedó tan disgustado que casi no pudo dormir. El otro monstruo estaba también muy irritado y durmió muy mal. A la mañana siguiente el primer monstruo se sentía fatal después de la mala noche que había pasado.

Se acercó al agujero y gritó:

(c) Psy, Soc, \& Educ, 2013, Vol.5, №1 
M1: ¡Despierta, melón, que se acaba la noche!

M2: ¡No seas estúpido, cerebro de mosquito! ... Eso es que viene el día. (y agarró una piedra y la tiró por encima de la montaña).

M1: ¡Tienes muy mala puntería, palurdo! .... (Buscó otra piedra más grande y la lanzó).

M2: ¡No tienes nada que hacer, patán, peludo, narizotas! . . . (y arrojó un pedrusco que desmochó la cima de la montaña).

M1: ¡Eres un buñuelo de viento, rancio y pringoso! ... (El primer monstruo lanza una roca que desprendió otro pedazo de montaña).

M2: ¡y tú eres un alcornoque viejo y renegrido! (Le dio una patada a una piedra enorme).

$\mathrm{N}$ : A medida que avanzaba el día las piedras iban siendo cada vez más grandes y los insultos cada vez más largos. Los dos monstruos seguían ilesos, pero la montaña se iba desintegrando.

M1: ¡Eres un zoquete peludo, cebón, cabeza hueca y mandón!

$\mathrm{N}$ : Gritaba el primer monstruo tirando una roca gigantesca. Esa roca terminó por destruir lo que quedaba de montaña y los dos monstruos se vieron por primera vez. Esto ocurrió precisamente cuando empezaba una nueva puesta de sol.

M1: ¡Increíble! . . . Ahí llega la noche. Tenías razón.

M2: ¡Asombroso! Tenías razón, es que el día se va.

$\mathrm{N}$ : Se reunieron en medio del desastre que habían organizado contemplaron la llegada de la noche y la marcha del día.

M1: Pues ha sido bastante divertido.

M2: ¿Verdad que sí? ... ...... ¡Lástima de montaña!

- Finalizamos la actividad con una reflexión: Dos monstruos enfrentados por ver la misma realidad desde perspectivas opuestas llegan a entenderse tras hacer desaparecer en una riña la montaña que los 
divide, y les impide ver las cosas de la misma forma. Comenta con los compañeros si alguna vez habéis discutido como esos monstruos, diciendo los dos lo mismo, pero con palabras distintas. Hablamos de los insultos y de la agresividad. Proponemos diferentes formas de resolver el conflicto de los dos monstruos. Pedimos de nuevo dos voluntarios que vuelvan a representar el cuento pero de forma amistosa.

Tabla 8. Actividad 6. Ponte en mi Lugar

\section{ACTIVIDAD 6: PONTE EN MI LUGAR}

- Introducción temática: movimiento y desplazamiento.

- Trabajamos la empatia a través de diferentes roles.

- Desarrollo: Comenzaremos la actividad pidiendo a los alumnos que se sitúen en un extremo del patio y les pediremos que lleguen al extremo contrario corriendo. Repetiremos el ejercicio una segunda vez, pero caminando $y$ moviéndose libremente. A continuación, dividiremos la clase en 3 grupos:

-El primer grupo serán aquellos que intentan llegar al otro extremo del patio caminando. Podemos llamarles "los caminantes".

-El segundo grupo, serán unos compañeros que intentan impedir que lleguen. Podemos llamarles "los muros".

-El tercer grupo, serán "los espectadores"; simplemente observarán la situación y algunos de ellos se reirán histriónicamente apoyando a "los muros".

Pediremos al primer grupo que intente llegar al otro extremo del patio simplemente intentando esquivar a "los muros" mientras éstos intentan frenarles. Al finalizar invertiremos los papeles tantas veees sea posible, hasta que todos los alumnos hayan pasado por los tres roles.

- Cuando hayan terminado, les reuniremos y les pediremos que digan si les ha resultado facil o dificil, como se han sentido y si les hubiera gustado que alguien les hubiera ayudado. También preguntaremos a los espectadores que sentian mientras vefan a sus compañeros. Repetiremos de nuevo la situación pero esta vez "los caminantes" tendrán la ayuda de "los espectadores", para poder avanzar. Se trata de hacer comprender a los alumnos, la importancia de ayudar a los demás en situaciones dificiles.

Tabla 9. Actividad 7. -¿Y tú qué Harías?

\section{ACTIVIDAD 9: Y TU ZOUÉ HARIAS?}

* Introducción temática: el teatro.

- Trabajamos la empatia y resolución de conflictos utilizando "El juego sobre el acoso escolar" (Berg, 2005).

- Desarrollo: proponemos una lluvia de ideas acerca de diferentes situaciones de conflicto que pueden surgir en el colegio o que hayan experimentado u observado recientemente con otros compañeros (te insultan, se rien de ti, te quitan o rompen algo. ven como le pegan a un compañero. etc.). A 

acoso escolar" pero no hacemos mención directa a los roles: victima y agresor.

Para el desarrollo e implementación de este programa es necesario contar con la implicación de todo el centro educativo: Tutores de $3^{\circ}$, dirección del centro, equipos docentes y especialista de psicología y pedagogía. El rol del docente será siempre el de guía y orientador de los alumnos, gestionando el clima del aula a lo largo del desarrollo de las actividades.

Cabe señalar el apoyo de las familias es fundamental. éstas deben ser informadas en todo momento, antes, durante y después del proyecto. Así mismo, resulta conveniente programar reuniones con las familias al menos una vez al mes, para realizar un seguimiento del trabajo realizado en clase por los alumnos. Sin embargo, si a lo largo del programa se detectara algún tipo de problema, se citaría a la familia de forma individual.

Para la evaluación de la intervención, pueden utilizarse instrumentos de observación para los docentes como los elaborados por Weir y Duveen (1981) o la adaptación de cuestionarios y escalas para el alumnado como los elaborados por Merhabian y Epstein (1972) y Ma y Leung, (1991). Por otro lado, Smith, Pepler y Rigby (2004) ofrecen una recopilación de estudios que evalúan mediante escalas, la aplicación de programas de intervención en el acoso escolar a nivel internacional. Así mismo, brindan importantes recomendaciones a la hora de diseñar la evaluación de programas de intervención. Entre ellas se encuentran: descartar en las explicaciones los factores irrelevantes, cuidar la validez interna o comparar grupos de la misma edad ya que los cambios en el acoso escolar están muy vinculados a ésta.

\section{CONCLUSIONES}

La intención de este estudio es elaborar una propuesta de intervención, que trabaje la conducta prosocial y más concretamente la empatía, a través de un programa de actividades sencillas y al alcance de cualquier docente, que puedan ser trabajadas transversalmente al currículo de Primaria. Se trata de prevenir el acoso escolar desde la educación de la competencia socio-emocional del grupoclase, sin aludir directamente a los roles de víctima y agresor para no reforzar su existencia. En los diferentes programas de prevención-intervención, ambos roles suelen estar muy diferenciados, y el trabajo principal suele estar centrado en ellos, dando la sensación que se pierden de vista la vertiente que más fuerza presenta para prevenir el problema: los espectadores, entendiendo éstos como todos aquellos que participan directa o indirectamente en el acoso escolar, sin ser víctima o agresor.

Tras la lectura de diversos estudios se constata que el desarrollo emocional constituye un aspecto importante de la personalidad y la escuela es uno de los principales espacios para su desarrollo, ya que "el periodo escolar se considera 
fundamental en la vida del ser humano, pues las actitudes radicales se adquieren en la infancia mediante la configuración de las convicciones" (Perea, 2002, p.32) y los alumnos pasan mucho tiempo en ella. Lo que allí ocurra contribuirá a configurar en gran parte su personalidad, y es por ello que la escuela es un lugar donde debe favorecerse la amistad y las relaciones con el grupo de iguales, potenciando la convivencia entre los alumnos a través de actividades que trabajen las competencias emocionales, dentro de las cuales se encuentra la empatía. El trabajo de la conducta prosocial, está directamente vinculado con las competencias sociales, y con la mejora de la convivencia en el aula: "la conducta prosocial debe ser considerada como una dimensión de la competencia social y que juega un papel importante en el desarrollo de las relaciones con los iguales" (zahn-Waxler y Smith, 1992, p.230).

Esta propuesta está centrada fundamentalmente en el alumnado, no obstante, no perdemos de vista la importancia que tiene el trabajar conjuntamente con el centro escolar, las familias y la sociedad en general, ya que éstos también tienen un impacto importante sobre la educación emocional. Es necesario que los centros escolares cuenten con unas políticas educativas que apoyen a las víctimas de acoso escolar, ya que en muchas ocasiones éstas tienen que abandonar los centros, convirtiéndose en víctimas dos veces: por el agresor y por la propia institución educativa.

Así mismo, para un mayor impacto el programa debería desarrollarse durante todo el curso e incluso a lo largo de toda la escolaridad. Sin embargo, el currículo de Primaria marca un ritmo de aprendizaje, dentro del cual resulta complicado incorporar otros contenidos necesarios en la educación. Es por ello que este programa contempla la aplicación transversal como la mejor forma de trabajo ya que pueden ser incluidos objetivos para trabajar las emociones y las habilidades sociales. Además, cabe destacar que no resulta necesaria una inversión en materiales sino que con una buena dosis de creatividad, pueden elaborarse actividades con tal fin.

Como señalan del Barrio et al. (2011, p.8): "En la gran mayoría de estudios se reconoce la necesidad de mejorar el clima socioemocional de los centros escolares de todos los niveles educativos reduciendo el acoso y la exclusión social",

\section{REFERENCIAS}

Andrés, S. y Barrios, A. (2006). De la violencia a la convivencia en la escuela: El camino que muestran los estudios más recientes. Revista Complutense de Educación, 20(1), 205-227.

Andrés, S. y Barrios, A. (2008). El modelo del alumnado ayudante a discusión: la opinión de los alumnos participantes y sus beneficiarios. Revista Electrónica de Investigación Psicoeducativa, 9, 4(2), 311-332.

Avilés, J. Mª (2003). El maltrato entre escolares en el contexto de las conductas de acoso. Bullying en la escuela. Modelos de intervención. En J. M. Avilés 
(coord.), Riesgos psicosociales en la enseñanza (7) Valladolid: Confederación de STEs-intersindical.

Avilés, J.Ma . (2006). Bullying: el maltrato entre iguales. Agresores, víctimas y testigos en la escuela. Salamanca: Amarú.

Avilés, J.M. y Monjas, I. (2005). Estudio de la incidencia de la intimidación y el maltrato entre iguales en la educación secundaria obligatoria mediante el cuesitonario CIMEI. Anales de Psicología, 21(1), 27-41.

Berg, B. (2005). El Juego Sobre el Acoso Escolar. Madrid: TEA Ediciones.

Bolado, A., y Gómez, R. (2005). Estudio de incidencia del maltrato entre iguales en cuatro centros de la ESO de Cantabria. En Mª I. Fajardo, F. Vicente, A. V. Díaz, I. Ruiz y J. A. del Barrio (Comps.). Aportaciones psicológicas y mundo actual (pp. 149-160). Badajoz: PSICOEX.

Currie C. et al (eds.) 2004. Young People's Health in Context: international report from the HBSC 2001/02 survey. WHO Policy Series: Health policy for children and adolescents Issue 4, WHO Regional Office for Europe, Copenhagen.

Currie C. et al (eds.) (2008). Inequalities in young people's health: international report from the HBSC 2006/06 survey. WHO Policy Series: Health policy for children and adolescents Issue 5, WHO Regional Office for Europe, Copenhagen.

Del Barrio, C., Barrios, A., Granizo, L., van der Meulen, k., Andrés, S. y Gutiérrez, H. (2011). Contribuyendo al bienestar emocional de los compañeros: evaluación del Programa Compañeros Ayudantes en un instituto madrileño.

European Journal of Education and Psychology, 4(1), 5-17.

Del Rey, R. y Ortega, R. (2001). Programas para la prevención de la violencia escolar en España: la respuesta de las Comunidades Autónomas. Revista Interuniversitaria de Formación del Profesorado, 41, 133-145.

Defensor del pueblo y UNICEF (2006). Informe "Violencia Escolar: El maltrato entre iguales en la Educación Secundaria Obligatoria 1999-2006”. Madrid: Documentos Defensor del Pueblo.

Díaz-Aguado, M.J. (2006a). Del acoso escolar a la cooperación en las aulas. Madrid: Pearson.

Díaz-Aguado, M.J. (2006b). Sexismo, violencia de género y acoso escolar. Propuestas para una prevención integral de la violencia. Revista de Estudios de Juventud, 73, 38-57.

Díaz-Aguado, M. J., Martínez Arias, R. y Martín, J. (2010). Estudio estatal de la convivencia escolar en la Educación Secundaria Obligatoria. Madrid: Ministerio de Educación.

Eisenberg, N. y Fabes, R. (1998). Prosocial development. En Damon, W, y Eisenberg, N. (eds.). Handbook of child Psychology. Social, emotional and personality development. New york: John Wiley.

Fuentes, M.J. (2005). Compartir sentimientos. Fernández, P. y Ramos, N. (Eds.). Corazones Inteligentes (269-288). Barcelona: kairós, 
Garaigordobil, M. (2005). Conducta antisocial durante la adolescencia: Correlatos socio-emocionales, predictores y diferencias de género. Psicología Conductual, 13 (2), 197-215.

Gómez Bahillo y cols. (2006). Las relaciones de convivencia y conflicto escolar en los centros educativos aragoneses de enseñanza no universitaria. Gobierno de Aragón. Departamento de Educación, Cultura y Deporte.

Gómez, A., Gala, F.J., Lupiani, M., Bernalte, A., Miret, M.T., Lupiani, S. y Barreto, M.C. (2007). El "bullying" y otras formas de violencia adolescente. Cuadernos de Medicina Forense, 13, 165-177.

González Portal, Mª.D. (1992). Conducta prosocial: Evaluación e Intervención. Madrid: Morata.

González, J.A., Álvarez, D., Rodríguez, C., González, P., Núñez, J.C. y Álvarez, L. (2010). Situación actual de la convivencia escolar en la educación secundaria. En Gázquez, J.J. y Pérez, M.C. (2010). Investigación en Convivencia Escolar. Variables Relacionadas (119-123). Granada: Grupo Editorial Universitario.

Górriz, A.B. (2009). Roles Implicados en el Acoso Escolar: Comprensión de la Mente, Maquiavelismo y Evitación de la Responsabilidad. Tesis Doctoral, Facultad de Ciencias Humanas y Sociales, Universitat Jaume I de Castellón, Comunidad Valenciana, España. Consultada en:

http://www.tdx.cat/bitstream/handle/10803/10530/gorriz.pdf?sequence=1

Greener, S. y Crick, N. (1999). Normative beliefs about prosocial behavior in middle childhood: what does it mean to be nice? Social Development, 8(3), 349-363.

Guijo, V. (2002). Estudio Multifactorial de la Conducta Prosocial en Niños de Cinco y Seis años. Tesis Doctoral, Departamento de Ciencias de la Educación, Universidad de Burgos, España. Consultada en: http://dspace.ubu.es:8080/tesis/bitstream/10259/60/1/Guijo_Blanco.pdf

Hoffman, M.L. (2000). Empathy and Moral Development. Implications for care and justice. New york: Cambridge University Press.

Iborra, I., Rodríguez, A., Serrano, A. y Martínez, P. (2011). Informe Situación del menor en la Comunidad Valenciana: víctima e infractor. Serie 18 documentos. VIU (Valencian International University), Centro Reina Sofía y Generalitat Valenciana.

Jiménez, A. (2007). Las conductas de acoso en el Primer Ciclo de Secundaria en la provincia de Huelva: un estudio ecológico. En J. J. Gázquez, M. C. Pérez, A. J. Cangas y N. yuste (Eds.). Situación actual y características de la violencia escolar (pp. 19-24). Granada: Grupo Editorial Universitario

Latané, B. y Darley, J. M. (1970). The unresponsive bysander: Why doesn't he help. New york: Appleton-Century

Leganés, E. y Pérez-Aldeguer, S. (2012). Un análisis cualitativo sobre el uso de la música en los libros de texto de inglés en Primaria. Tejuelo, 13, 102-122.

Ma, H. k., y Leung, M. C. (1992). Effects of age, sex and social relationships on the altruistic behavior of Chinese children. Journal of Genetic Psychology, 153(3), 293-303. 
Masnou, F. (1994). Prosociabilidad. En M. Martínez y J. Puig (Coord.). La educación moral. (pp. 151-160). Barcelona: Graó. 2a Edición.

Mehrabian, A. y Epstein, N. (1972). A measure of emotional empathy. Journal of Personality, 40, 525-543.

Mora-Merchán, J.A. (2000). El fenómeno bullying en las escuelas de Sevilla. Tesis Doctoral no publicada, Departamento de Psicología Evolutiva de la Educación, Universidad de Sevilla, España.

Olweus, D. (1998). Conductas de acoso y amenaza entre escolares. Madrid: Morata.

Olweus, D. (2001). Peer harassment. A critical analysis and some important issues. En J.

Juvonen, y S. Graham (Eds.) Peer harassment in school (3-20). New york: Guilford Publications.

Organización Mundial de la Salud (2003). Informe mundial sobre la violencia y la salud. Ginebra: OMS.

Ortega, R. (1999). Los derechos de los niños y las niñas y la violencia entre iguales en el ámbito escolar. Informe SIAS 2. Sevilla: Asociación Española de Pediatría, pp.115.124.

Ortega, R., Romera, E. y Del Rey, R. (2009). Protagonismo de la atención a la convivencia en la prevención de la conflictividad y la violencia escolar. Informació Psicològica, 95, 4-14.

Patterson, G.R., Littman, R.A. y Bricker, W. (1967). Assertive behavior in children: A step towards a theory of aggression. Monographs of the society for research in child development, 35(5).

Patterson, G.R., Shaw, D.A. y Ebner, M.J. (1969). Teachers, peers and parents as agents of change in the classroom. En F.A.M. Benson (Ed.). Modifying deviant social behaviors in various classroom settings. Oregón: Departamento de Educación Especial.

Perea, R. (2002). Educación para la salud, reto de nuestro tiempo. Educación $X X I, 4,15-40$.

Sáinz, A., Angulo, A., Elorza, C. y Méndez, y. (2008). El maltrato entre iguales en Euskadi. Asturias: Instituto Vasco de Evaluación e Investigación Educativa.

Salmivalli, C, Lagerspetz, k., Björkqvist, k., Österman, k., kaukiainen, A. (1996). Bullying as a group process: Participant Roles and their relations to Social Status Within the group. Aggressive Behavior, 22, 1-15.

Salmivalli, C. (1999). Participant role approach to school bullying: implications for interventions. Journal of Adolescence, 22, 453-459.

Serrano, A. (2006). Acoso y Violencia en la escuela. Como detectar, prevenir y resolver el bullying. Barcelona: Ariel.

Serrano, A. e Iborra, I. (2005). Informe Violencia entre compañeros en la escuela. Valencia: Centro Reina Sofía para el Estudio de la Violencia.

Smith, P.k., Pepler, D. y Rigby, k. (Eds.) (2004). Bullying in schools. How successful can interventions be? Cambridge: Cambridge University Press. 
Solomon, R.W. y Wahler, R.G. (1973). Peer reinforcement of classroom problem behavior. Journal of Applied Behavior Analysis, 6, 49-56.

Staneva, z. y Molhova, M. (2011). The bullying phenomenon and the "I am not scared" project in Bulgaria or how and why we need to influence the national school-education system. En Psychology, Society, \& Education, 3(2), 147157.

Weir, k. y Duveen, G. (1981). Further development and validation of the prosocial behavior questionnaire for the use by teachers. Journal of Child Psychology and Psychiatry, 22, 357-374.

zahn-Waxler, C. y Smith, D. (1992). The development of prosocial behavior. En V. Van Hasselt y H. Michel (eds.). Handbook of social development: A lifespan perspective. Perspectives in developmental psychology (pp. 181188). New york: Plenum Press.

Bully Dance. Collell y Escudé. Extraído el 23 de Noviembre de 2011 desde http://www.xtec.es/ jcollell/z94Bully\%20Dance.pdf

Dilemas morales. Extraído el 5 de Diciembre de 2011 desde http://hazloquedebas.wikispaces.com/Dilemas+morales

Recursos sobre empatía. Extraído el 5 de Diciembre de 2011 desde http://www. xtec.es/ jcollell/z07Materials.htm

Servicio de prevención y ayuda contra el acoso escolar. Guía para el profesorado sobre el acoso escolar: detección, identificación, intervención y prevención. Gobierno de Canarias: Dirección general de ordenación e innovación educativa. Extraído el 23 de Octubre de 2011 desde http://www.gobcan.es/educacion/dgoie/publicace/docsup/guia_acoso.pdf 